\title{
Se maintenir à flot
}

\author{
Citation : CMAJ 2021 June 14;193:E917-8. doi : 10.1503/cmaj.210514-f
}

Voir la version anglaise de l'article ici : www.cmaj.ca/lookup/doi/10.1503/cmaj.210514

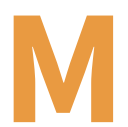

on fils du milieu, Matthew, s'est récemment inscrit au cours Étoile de bronze de la Société royale de sauvetage du Canada. À 10 ans, il était le plus jeune de son groupe, dont faisaient également partie son frère et son cousin. L'une des exigences du cours consistait à pouvoir nager pendant une minute en tenant une brique de 10 livres. Jusqu'au tout dernier cours, Matthew en a été incapable; il a même échappé la brique à une occasion, et c'est son cousin quelque peu exaspéré qui a dû aller la récupérer au fond de la piscine. Puisque je ne pouvais pas entrer dans l'enceinte de la piscine en raison de la COVID-19, j'ai fait promettre à mon plus vieux d'encourager son petit frère. Alors que je conduisais les trois garçons à leur dernier cours, les conseils fusaient dans la voiture.

«Tu dois garder ton menton hors de l'eau et battre des jambes sans arrêt. Et peu importe ce que tu fais, ne lâche pas la brique, même si elle est trop lourde, parce que personne n'ira la chercher au fond de la piscine », l'a averti son frère.

"Aussitôt que ton temps sera écoulé, on va te sortir de l'eau », a ajouté son cousin.

"J'essaie », a répliqué Matthew, «mais j'ai l'impression de me noyer et on dirait que ça prend une éternité avant que le sauveteur donne son coup de sifflet... »

Ce commentaire m'interpelle énormément. En tant que chirurgienne oncologue, je porte ma lourde charge à moi, et je le fais depuis le début de ma pratique en 2008. J'ai la responsabilité de guider mes patients tout au long de leur combat contre le cancer, du diagnostic à la stadification du cancer, à la résection chirurgicale, aux soins postopératoires et, enfin, à l'orientation vers un autre oncologue pour des

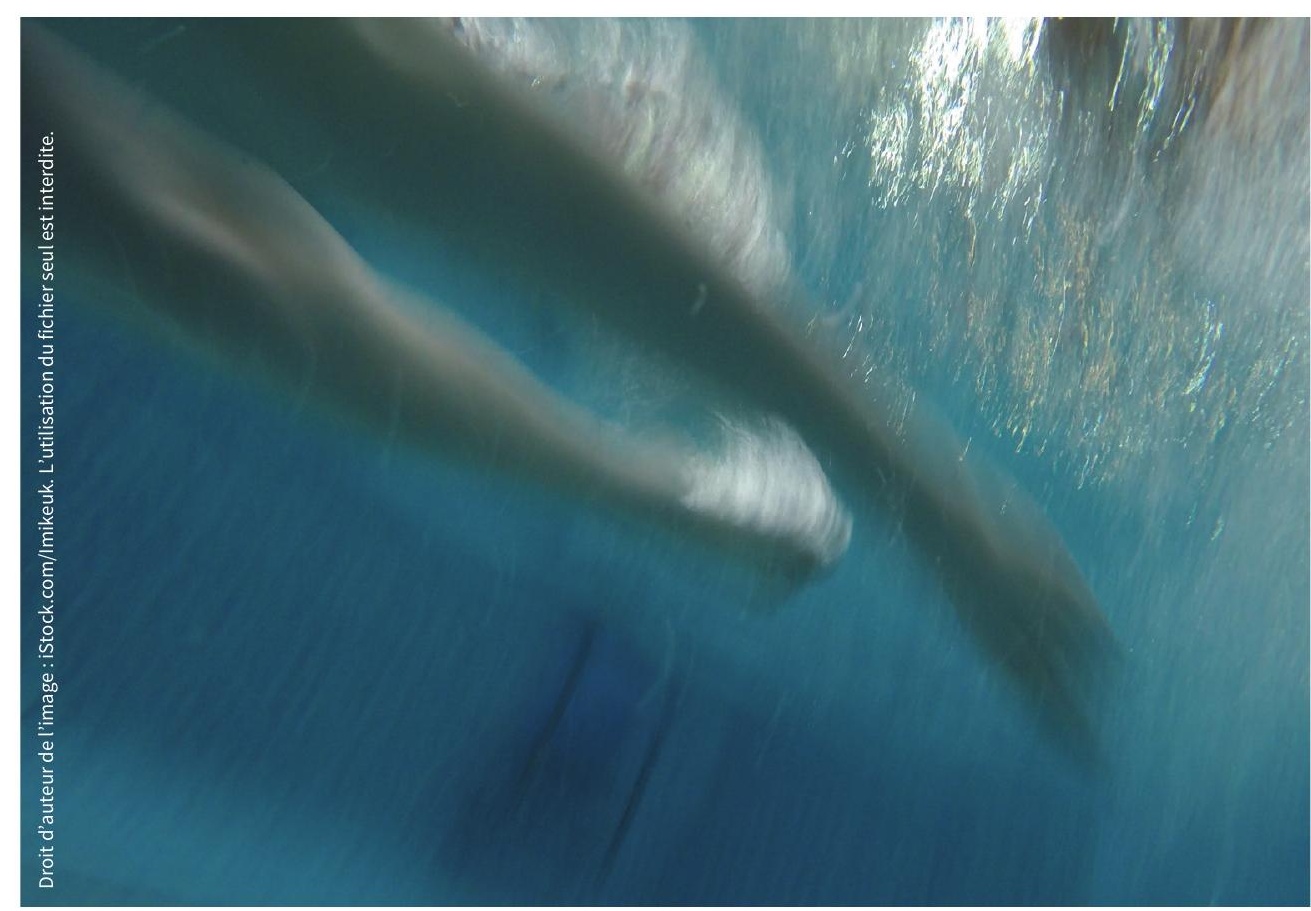

traitements supplémentaires ou à l'inscription à un programme pour les survivants. Je ne vois pas cette responsabilité comme une brique, mais plutôt comme un sac à dos rempli de pierres précieuses, comme des diamants. Chacune de ces pierres représente un patient ou une patiente qui a besoin de moi pour traverser cette épreuve en toute sécurité, de manière efficace et avec compassion.

Mon sac à dos m'accompagne toujours et, même si je le pose par terre de temps à autre, il n'est jamais très loin. Ma famille ne le connaît que trop bien. Quand ce n'est pas le laboratoire qui m'expédie des résultats sanguins alarmants en pleine soirée cinéma, c'est le radiologue qui m'appelle au sujet de résultats de tomodensitométrie préoccupants durant un match de soccer, ou encore une patiente en soins postopéra- toires qui m'envoie un message texte juste avant un récital de piano parce qu'elle est fiévreuse. Je dois alors arrêter ce que je suis en train de faire, trouver mon sac à dos et le ramasser. Les dossiers médicaux électroniques de mes patients sont liés à mon iPhone, ce qui fait que je reçois des notifications quand de nouveaux résultats arrivent ou qu'un fournisseur de soins veut me poser une question. Je reçois même une alerte sur ma montre Apple Watch pour les messages cliniques importants.

Pourtant, même s'il exige une attention constante, je n'ai jamais éprouvé de ressentiment envers mon sac à dos. La prise en charge de mes patients fait partie intégrante du serment que j'ai prêté pendant mes études en médecine il y a des années, et le fait d'être là pour eux est ce qui rend ce travail si satisfaisant. C'est un privilège que de pouvoir les aider, et c'est 
la raison pour laquelle la dernière année, avec la pandémie de COVID-19, a été indescriptiblement difficile.

Étant chirurgienne oncologue, je n'ai pas eu à prendre de décisions quant à la distribution des respirateurs ou à l'attribution des lits de soins intensifs, mais les contraintes imposées par la pandémie en matière de ressources sont insidieuses, chroniques et constantes; elles érodent lentement ma résilience et mon courage. Derrière chaque biopsie retardée de plusieurs semaines, voire de plusieurs mois, derrière chaque tomodensitométrie qui ne peut pas avoir lieu parce que la situation n'est pas suffisamment prioritaire, derrière chaque chirurgie annulée ou retardée à cause de la pression exercée sur notre système de soins de santé déjà surchargé se trouve un patient ou une patiente - un diamant dans mon sac à dos. Chaque fois que je dois annoncer à une personne un diagnostic de cancer alors qu'elle est assise seule dans mon bureau parce que sa famille ne peut pas l'accompagner, je sais qu'elle mérite mieux. Chaque fois que je dois rassurer un membre de la famille au téléphone, lui dire que nous faisons tout ce que nous pouvons malgré les compli- cations graves et que je voudrais tant lui permettre d'être au chevet de l'être aimé, je partage sa frustration. Chaque fois trop souvent - , j'ai l'impression de le laisser tomber. Au cours des 12 derniers mois, j'ai passé de nombreuses heures à revendiquer, à plaider, à implorer, à supplier et même parfois à pleurer pour obtenir les ressources dont j'ai besoin pour prendre soin de mes patients atteints du cancer. Et je ne suis pas la seule. Les autres médecins, infirmières et administrateurs de mon hôpital travaillent tout aussi fort et trouvent la situation tout aussi pénible, mais ça ne rend pas les choses plus faciles.

Depuis le début de la pandémie, le poids de mon sac à dos est le même, mais à présent, c'est comme si je devais nager avec lui sur le dos. Au début, je nageais avec vigueur, mes jambes battaient l'eau à toute vitesse et je gardais la tête hors de l'eau. Nous étions tous dans le même bateau et j'étais déterminée, convaincue que nous allions nous en sortir. Aujourd'hui, alors que le pic d'une autre vague de COVID-19 limite davantage les ressources pour mes patients atteints du cancer, je sens le poids me tirer sous l'eau. Mes jambes sont épuisées et c'est à peine si j'arrive à garder le menton hors de l'eau. Si je laisse tomber le sac à dos, il coulera au fond de la piscine, avec les conséquences dévastatrices que cela suppose. Mais en ce moment, j'ai l'impression de me noyer.

Je sais que je ne suis pas seule. Nous sommes nombreux à essayer de nous maintenir à flot. Je ne sais pas quoi faire d'autre que de continuer de battre des jambes, de respirer et d'espérer que le sauveteur donne son coup de sifflet pour que nous puissions enfin nous sortir mutuellement de l'eau.

\section{Rebecca Ann C. Auer MD MSc}

Département de chirurgie, L'Hôpital d'Ottawa, Ottawa, Ont.

Cet article a été révisé par des pairs.

Propriété intellectuelle du contenu : Il s'agit d'un article en libre accès distribué conformément aux modalités de la licence Creative Commons Attributions (CC BY-NC-ND 4.0), qui permet l'utilisation, la diffusion et la reproduction dans tout médium à la condition que la publication originale soit adéquatement citée, que l'utilisation se fasse à des fins non commerciales (c.-à-d., recherche ou éducation) et qu'aucune modification ni adaptation n'y soit apportée. Voir : https://creativecommons.org/ licenses/by-nc-nd/4.0/deed.fr. 